\title{
1 Universality of the developmental origins of diverse 2 functional maps in the visual cortex
}

3 Min Song ${ }^{1,2 \dagger}$, Jaeson Jang ${ }^{1 \dagger}$, Gwangsu $\operatorname{Kim}^{3}$ \& Se-Bum Paik ${ }^{1,2 *}$

$4 \quad{ }^{1}$ Department of Bio and Brain Engineering, ${ }^{2}$ Program of Brain and Cognitive Engineering,

$5 \quad{ }^{3}$ Department of Physics, Korea Advanced Institute of Science and Technology, Daejeon

6 34141, Republic of Korea

$7 \quad+$ These authors contributed equally to this work

8 *Correspondence and requests for materials should be addressed to Se-Bum Paik (spaik@kaist.ac.kr).

10 Abstract

11 The primary visual cortex of higher mammals is organized into diverse functional maps of

12 correlated topography, implying an efficient tiling of functional domains. However, no

13 fundamental principle is available on how systematic organization of the maps could

14 develop initially in various species. Here, we propose that diverse functional maps are

15 seeded from a common framework of retinal afferents, and that this universality of

16 development explains the observed topographical correlations among the maps. From the

17 simulation of retinal ganglion cell mosaics, we successfully developed all four cortical

18 maps observed so far. We validated our model prediction of inter-map relationships from

19 analysis of map data in evolutionarily divergent mammalian species. Our key prediction,

20 the hexagonal periodicity of every functional map, was validated from analysis of the map

21 data in diverse mammalian species. Our results provide new insight into a universal

22 mechanism for the development and evolution of the visual cortex. 


\section{Introduction}

24 In higher mammals, the primary visual cortex (V1) is organized into various functional 25 maps of neural tuning such as ocular dominance ${ }^{1}$, preferred orientation ${ }^{2}$, direction ${ }^{3}$ and spatial frequency ${ }^{4}$. Although the role of each functional map in visual information processing is under debate ${ }^{5,6}$, correlations between the topographies of different functional maps have been observed, implying their systematic organization. For instance, it was reported that the gradient of orientation tuning intersects orthogonally with that of ocular dominance $^{7}$ and preferred spatial frequency ${ }^{8}$ in the same cortical area. High-resolution two-

31 photon imaging data revealed that the region of higher spatial frequency tuning tends to

32 align with the binocular region in the ocular dominance map ${ }^{9}$. Such structural correlation 33 between the maps is thought to achieve a uniform representation of visual features across 34 cortical areas ${ }^{10}$. In addition, these findings in phylogenetically distinct mammalian species imply that there may exist a universal principle of development and evolution organizing individual functional maps ${ }^{11}$, possibly which results in efficient tiling of sensory modules.

Important clues regarding the development of the maps were found in the thalamic origin of the selective response in V1. When cortical recurrent activity was silenced so that only thalamic afferents were provided, the preferred orientation angle of a V1 neuron remained consistent ${ }^{12,13}$, which suggests that cortical orientation tuning originates from

41 feedforward afferents. In addition, it was reported that orientation tuning in V1 is predictable from the local average of thalamic ON and OFF receptive fields ${ }^{14}$. At larger scales, it was reported that the topography of functional maps in V1 is strongly correlated with the spatial arrangement of thalamic afferents ${ }^{15,16}$. Furthermore, it was recently observed that direction preference in V1 arises from temporal delay in thalamic inputs ${ }^{17}$.

46 These observations altogether imply that functional tuning in the cortical neurons may 
originate from a common retinal or thalamic afferent (Fig. 1a); thus map structures of various functional tuning might be seeded initially from the same blueprint of feedforward projections.

Given this, how could the feedforward afferents provide a regularly structured layout of functional tuning to seed an orderly architecture of the map? The statistical wiring model $^{18,19}$ suggested that V1 receptive field structure is constrained by the local structure of $\mathrm{ON}$ and OFF mosaics of retinal ganglion cells (RGCs $)^{20}$. In this view, the receptive field of a V1 neuron is generated from the sum of receptive fields of local RGC that provides feedforward afferents, and the V1 neuron is tuned to orientation due to the anisotropy of the receptive fields. Adapting this notion, the moiré interference model further proposed that spatial interference patterns between the hexagonal mosaics of ON and OFF RGC generate a quasi-periodic pattern that seeds the topography of an orientation map early in development ${ }^{21,22}$ (Fig. 1b-c). The key assumption that RGC mosaics patterns seed the topography of cortical orientation maps, allowed this model to successfully explain how regularly structured cell mosaics in the retina could seed the early structure of an orientation map ${ }^{23}$ and its hexagonal periodicity ${ }^{24}$ in diverse species of higher mammals.

Herein, expanding this developmental principle of orientation maps, we propose that such a regular distribution of RGCs can also induce multi-modal functional maps in V1 (Fig. 1d-g). Our hypothesis is that local ON and OFF afferents can induce multiple types of neural tuning according to their spatial and temporal profiles. First, temporal delay between $\mathrm{ON}$ and $\mathrm{OFF}$ pathways induces direction selectivity in receptive fields of $\mathrm{ON}$ and $\mathrm{OFF}$ subregions (Fig. 1d). Second, variation of the distance between neighbouring ON and OFF RGCs modulates the degree of overlap between ON and OFF receptive field subregions, which varies the sum of neural responses to ON and OFF stimulus in contralateral 
71 afferents. This induces competition between contra- and ipsilateral afferents, resulting in a periodic pattern of ocular dominance (Fig. 1e). Lastly, in the binocular region, intermingling of both contra- and ipsilateral inputs with different spatial phase of receptive field induces a preference to higher spatial frequency than that in the monocular region (Fig. 1f). Based on this model, simulations were able to reconstruct successfully each functional map and the observed topographical correlations between them (Fig. 1g).

We validated our model predictions using published animal data on evolutionarily divergent species ${ }^{7,10,25-30}$. We confirmed that all the predicted relationships between the local structures of different functional maps from the model were observed in experimental data. In addition, our new prediction that iso-domains of feature preference are arranged in

81 a hexagonal lattice in each type of functional map was confirmed using map data from various species. Taken together, we suggest that the regularly structured retinal afferents provide a common framework of organizing various functional maps in the visual cortex.

We propose a new theory that the layouts of cortical functional maps are seeded from the spatial distribution of ON and OFF ganglion cells in the retinal mosaics (Fig. 1). To validate this model, first we performed a series of computer simulations using an ideal model of retinal mosaics where the locations of ON and OFF receptive fields lie at the vertices of hexagonal lattices ${ }^{31}$ (Fig. 1b). In our previous study ${ }^{21}$, the model showed how a single pair of ON and OFF RGC mosaics from the contra-lateral eye could initially generate the structure of orientation maps ${ }^{23}$ (Fig. 1c). In the current study, we expand this model to consider both contra- and ipsilateral afferents to V1, to investigate the emergence 
94 of other functional maps. Our results show that retinal afferents could induce periodic patterns of direction preference (Fig. 1d, Fig. 2), ocular dominance (Fig. 1e, Fig. 3) and spatial frequency preference (Fig. 1f, Fig. 4), from the regularly structured moiré interference pattern of RGC mosaics.

\section{Direction tuning discontinuity in the iso-orientation tuning domain}

100 If the local alignment of ON and OFF retinal inputs constrains orientation tuning (Fig. 1c),

101 how does direction tuning arise from these retinal afferents? We hypothesized that

102 difference in temporal response profiles between ON and OFF RGCs could induce the

103 direction tuning of V1 neurons. From the observed delay of response kernels between ON

104 and OFF RGCs afferents ${ }^{32}$ (Fig. 2a), a V1 neuron connected to them would have a

105 spatiotemporally non-separable receptive field (Fig. 2b), and would induce direction tuning

106 (Fig. 2c). This asymmetric spatiotemporal receptive field generates a selective response to

107 the stimulus that drifts from ON to OFF RGCs (Fig. 2d), resulting in a preferred direction

108 angle in the ON-to-OFF direction (Fig. 2e).

Such common origin of orientation and direction tuning, explains the topographical

110 correlation between the orientation and direction maps. The iso-orientation domains of an

111 orientation map exhibit substantial overlap with the linear fractures of a direction map, in

112 which the preferred direction suddenly flips to the opposite direction ${ }^{27}$. From the notion that

113 the preferred direction of a V1 neuron is constrained by the direction of the ON-to-OFF

114 subregion of a receptive field (Fig. 2e), our model predicts that a moiré interference pattern

115 of ON and OFF RGC mosaics contains linear fractures of ON-OFF direction, forming

116 preferred direction discontinuities (Fig. 2f). In this view, direction discontinuities would 
117 appear in the middle of the iso-orientation domain (Fig. 2g-i). To validate this model

118 prediction, we examined previously reported map data of cats $^{27}$, to see if the iso-orientation

119 domains of orientation maps overlap with the direction fractures of the direction map. We

120 confirmed that the overlap ratio observed in the map data was significantly greater than

121 chance (Fig. 2 j, model: $0.109 \pm 0.022, N=100$, data: $0.106, N=1$, randomly shifted data:

$1220.05 \pm 0.005, N=100$; model vs. shifted: $P<10^{-15}, T=-25.723$, two-tailed t-test; data vs.

123 shifted: $P<10^{-15}, T=-126.15$, one sample two-tailed t-test; model vs. data: $n . s ., P=0.177$,

$124 T=1.36$, one sample two-tailed t-test), suggesting that orientation and direction maps share

125 a common substrate of spatial organization. On the other hand, when we spatially shifted

126 the direction map from the orientation map and measured again, the overlap ratio

127 noticeably decreased as the amount of shift increased, confirming that the predicted

128 correlation between the two maps does exist (Fig. 2k).

Development of ocular dominance with RGC mosaics from two eyes

131 We showed that a pair of ON and OFF RGC mosaics (the retinal afferents from the

132 contralateral eye) could seed the layout of orientation and direction maps. However, to

133 describe the development of ocular dominance, the inputs from the ipsilateral eye must be

134 considered as well. With a notion that thalamocortical projections from the contralateral

135 eye arrive at V1 ahead of those from the ipsilateral pathway ${ }^{33}$, we implemented this process

136 so that the connections from each pathway would develop sequentially (Fig. 3a). From the

137 simulation, we propose that the RGC mosaics of the contralateral retina also initiate

138 periodic topography of cortical response by contra- and ipsilateral afferents, and result in

139 the ocular dominance map. 
At early developmental stage when only the contralateral afferents are provided,

141 spatial variation of the ON-OFF distance in RGC mosaics could induce periodic fluctuation

142 of local cortical activity (Fig. 3b-c). When ON and OFF RGCs are close to each other, ON

143 and OFF subregions in the V1 receptive field largely overlap and cannot be simultaneously

144 activated by visual stimulus. In this case, the cortical responses from ON and OFF afferents

145 are not summed up at their peak values, resulting in weak total response (Fig. 3b, top, Fig.

146 3c, white circle). In contrast, when ON and OFF subregions are more separated, both

147 subregions can be simultaneously activated by proper stimuli, resulting in stronger cortical

148 response (Fig. 3b, bottom, Fig. 3c, white triangle). The estimated cortical response

149 appeared as an increasing function of the distance between ON and OFF RGCs. Moreover,

150 the moiré pattern of contralateral RGC mosaics could induce periodic cortical activity, even

151 without spatial variation in the feedforward connection strengths (Fig. 3c). Such a pattern

152 of cortical response at the early stage could provide a blueprint for the ocular dominance

153 map (Fig. 3d-f).

To simulate a complete developmental process for an ocular dominance map, we first modelled activity-dependent wiring of the contralateral pathway ${ }^{33}$ such that the strength of

156 feedforward connection was potentiated as the response of a postsynaptic neuron to

157 feedforward spikes got stronger. (Fig. 3a; for details, see Methods). As a result, a two-

158 dimensional hexagonal pattern of contralateral wiring strength was developed in the cortex

159 from the ON and OFF RGC mosaics structure (Fig. 3d and Supplementary Fig. 1). Next,

160 we allowed the same type of plasticity in the ipsilateral pathway and simulated the

161 development of cortical afferents from both eyes. To examine the balance between contra-

162 and ipsilateral afferents at unit scale, we normalized the total strength of contra- and

163 ipsilateral afferents for each V1 neuron as a constant. Thus neurons with strong

164 contralateral afferents received relatively weaker ipsilateral inputs and vice versa (Fig. 3e). 
165

166

As a result, this developmental process reproduced a periodic ocular dominance map, the layout of which was initially seeded by contralateral RGC mosaics (Fig. 3f).

A key prediction of our model is that the ocular dominance at a cortical site depends on the distance between $\mathrm{ON}$ and $\mathrm{OFF}$ receptive fields from the contralateral pathway. We were able to validate this prediction using recently published cat data of multi-electrode recordings across the $\mathrm{V}^{15}$ (Fig. 3g). We confirmed that the distance between ON and OFF afferents from the contralateral pathway, as well as the ocular dominance, varied periodically across the cortical surface and that their spatial periods were practically identical (only $0.7 \%$ different; Fig. $3 \mathbf{h}$ and i). In addition, the observed ocular dominance was significantly correlated with the distance between ON and OFF receptive fields from the contralateral pathway, but not with that from the ipsilateral pathway (Fig. 3h, ocular dominance vs. $d_{\text {contra: }} r=+0.602, P=0.002$, lag $=0.2$ cycle of ocular dominance period, ocular dominance vs. $d_{\text {ipsi: }} r=-0.441, P=0.605$, lag $=-0.5$ cycle). This result is consistent with our model prediction that the layout of the ocular dominance map is constrained by the spatial organization of contralateral afferents.

One might argue that the ocular dominance map in a monkey shows a stripe pattern, different from the blob-like pattern in cats and our simulation (Fig. 3f). The degree of anisotropy in the retinotopy may provide a clue to explain this difference across species. The focus of our simulation was on the condition of isotropic retinotopy, where magnification factors along the polar and eccentric axes are similar to each other, as observed in the $\mathrm{cat}^{34}$. However, in monkeys, the ratio between these two magnification factors is about $1.5^{35}$, implying that the same distance on each axis in the retinal space is noticeably different in the cortical space. When this anisotropy is introduced in our model, it distorted the initial blob-like pattern, so that blobs were connected along the polar axis, 


\section{Development of spatial frequency map from binocular afferents}

193 Next, we show that the ocular dominance patterns in the V1 can induce the organization of

194 spatial frequency maps. In the previous study, it was reported that the spatial organization

195 of frequency tuning in V1 neurons matches the structure of the ocular dominance map in a

196 way that the neurons of higher binocularity (receives strong inputs from both eyes) are tuned to higher spatial frequency ${ }^{9}$. Here, we show that this relationship between spatial

198 frequency tuning and binocularity is achieved from a common origin of the two maps, the spatial organization of ON and OFF afferents (Fig. 4). contra- or ipsilateral dominant region, depending on the distance between $\mathrm{ON}$ and OFF

202 (Fig. 4a). Thus, when the ON-OFF distance in the contralateral RGC mosaics is minimum and maximum, the corresponding V1 regions become monocular (either contra- or

204 ipsilateral dominant; Fig. 4b, top and bottom), while the areas in between become binocular 205 regions where the neurons receive balanced input from both eyes (Fig. 4b, middle). During 206 this process, we first found that the orientation preference of ipsilateral afferent can match 207 that of contralateral receptive field through an activity-dependent matching process

208 (Supplementary Fig. 1), as observed in animal data ${ }^{23}$. More importantly, we found that the 209 phase difference between the contra- and ipsilateral receptive fields ${ }^{36}$ induces a preference 210 to higher spatial frequency of the summed receptive field in binocular regions (Fig. $\mathbf{4 b}$, 211 middle). As a result, a periodic spatial frequency map is induced along with the ocular 
212 dominance map that is initially seeded from the contralateral retinal mosaics (Fig. 4c).

213 From our model simulations, we observed that the spatial frequency tuning was strongly

214 correlated with the binocularity in all local cortical areas (Fig. 4d, Pearson correlation

215 coefficient, $N=144, r=+0.548, P=1.082 \times 10^{-12}$ ). Again, we validated this model

216 prediction from analysis of the monkey data used previously ${ }^{9}$ (Fig. 4e, Pearson correlation 217 coefficient, $\left.N=164, r=+0.681, P<10^{-15}\right)$.

\section{Topographical correlations of orientation, direction, spatial frequency and ocular}

\section{dominance maps}

221 How can the observed correlations arise among the diverse functional maps in the cortex?

222 Here, we propose that the structure of retinal afferents provides a blueprint for every

223 functional map in the cortex, and this common origin of RGC mosaics induces intrinsic

224 structural correlations among the maps (Fig. 5a-d).

Previously, we showed that orientation pinwheels develop at the locations where the distance between RGC ON and OFF receptive fields is either local maxima or minima ${ }^{22}$

227 (Fig. 5a). If we examine other functional tunings of neurons around such singularities, it is 228 observed that the orientation, ocular dominance and spatial frequency tunings must have

229 well-correlated map structures, each with contribution from the structure of retinal ON and

230 OFF afferents (Fig. 5b). As illustrated in the model RGC mosaics (Fig. 5a), the distance

231 between the nearest ON and OFF RGC varies in the direction (Fig. 5a, red arrow)

232 orthogonal to that of ON-OFF dipole angle variation (Fig. 5a, blue arrow). Thus, the

233 gradient of a preferred orientation map changes in a circular direction around the

234 orientation pinwheels (Fig. 5b, left). At the same time, ocular dominance which depends on 
235 the distance between ON and OFF RGCs, changes in the radial direction from the

236 singularity (Fig. 5b, middle). As a result, gradients of the two maps must cross

237 orthogonally in the cortical space. Similarly, a spatial frequency map that develops parallel

238 to the ocular dominance map is aligned orthogonal to that of the orientation map (Fig. $\mathbf{5 b}$,

239 right).

We validated these model predictions using published functional map data from

241 animal experiments and confirmed the relationship between the maps observed in the

242 simulation. As predicted by our model, both ocular dominance (cat ${ }^{7,10,25,28,37}$, monkey ${ }^{9,38}$,

243 galago $^{26}$ ) and spatial frequency tuning maps ( cat $^{7,10,25}$, monkey $\left.{ }^{8,9}\right)$ showed orthogonal

244 relationship to the structure of the orientation map in the same area (Fig. 5c-d). Thus, we

245 concluded that our model, in which cortical functional maps develop universally from the

246 moiré interference patterns in RGC mosaics, could provide a plausible explanation for the

247 development of functional maps and their structural correlations.

\section{Hexagonal symmetry in diverse functional maps}

250 Another key prediction of our developmental model arises from the intrinsic features of

251 RGC moiré interference patterns: hexagonal symmetry. From the fact that $\mathrm{ON}$ and OFF

252 RGC mosaics are hexagonal lattices ${ }^{31}$ and thus also are their interference patterns ${ }^{39}$, our

253 model predicts that each functional map that develops from RGC afferents must have

254 hexagonal symmetry in the map topography in common. We tested this prediction using

255 published animal data on direction ${ }^{27,29,30}$, ocular dominance ${ }^{7,10,25,26,28}$ and spatial frequency

$256 \operatorname{maps}^{7,25}$ from different species as was done for orientation maps in our previous study ${ }^{21}$. 
As predicted, 2D autocorrelation of the individual maps showed a hexagonal pattern of peaks (Supplementary Fig. 3). The statistical significance of the local peaks was tested

259 against control maps that matched the spectral power and spatial periodicity of the original 260 map, but with an isotropic amplitude spectrum instead of hexagonal periodicity (for details,

261 see Supplementary Fig. 3 or reference 21). We observed that all the hexagonal

262 autocorrelation peaks of individual maps exhibited a significance level of $P<0.01$. In the

263 average plot of autocorrelations, we confirmed that the peaks closely matched the expected 264 hexagonal periodicity in three types of functional maps from species as different as cat, 265 ferret, and galago (Fig. 5e).

\section{Discussion}

How are various functional maps created? Is there a universal principle on the development of these functional architectures in the cortex? With these questions in mind, we introduced a novel model assuming that all the observed functional maps arise from projection of the

271 structure of retinal mosaics (Fig. 1), and that this common origin induces systematic

272 organization among the layouts of different maps, such as the orthogonal intersection of

273 map gradients. In addition, we found hexagonal arrangement of iso-domains in each

274 functional map by analysing the published animal data for various species, which has never

275 been reported previously. These results support our idea of a single universal principle 276 active in the developmental mechanism of diverse functional maps in the V1.

278 by our model. It has been reported that the ocular dominance map arises even before the

279 inputs from both eyes are segregated in the cortex ${ }^{23,40}$, and this observation is 
understandable because the initial arrangement of ocular dominance domains does not

281 require inputs from both eyes in our model. Considering the earlier development of the

282 contralateral visual pathways ${ }^{33}$, our model hypothesis that spatial variation of inputs from

283 the contralateral retinal mosaics is sufficient to induce the arrangement of the contralateral

284 dominant domains (Fig. 3d) seems to be biologically plausible and is consistent with earlier

285 observations of ocular dominance maps. The ipsilateral afferents are weak at this stage, so

286 the cortical response is strongly modulated by the contralateral eye during early

287 development ${ }^{23}$. Because the layout of an ocular dominance map can be determined by the

288 input from one eye, the spatial relationship between ocular dominance and the orientation

289 map is not ruined under monocular deprivation ${ }^{41}$.

The observed change in preferred frequency throughout the developmental stages is

291 also explainable by the model. The V1 neurons show preference for relatively lower spatial

292 frequency at the early stage of postnatal development, but preference tuning for higher

293 spatial frequency arises at later stages ${ }^{42}$. Our model explains that this is because the

294 selectivity for higher spatial frequency can develop when inputs from both eyes are

295 provided to V1 neurons ${ }^{9}$. The anatomical connections of ipsilateral pathways arrive initially

296 at V1 weeks before the observation of tuning for high frequency ${ }^{40}$. Effective connections

297 are likely to take several weeks to be refined to match the orientation preferences between

298 the two eyes for the complete development of the circuits ${ }^{23}$.

300 maps may have caused difficulty in precise analysis of the location of orientation pinwheels

301 on spatial frequency maps and resulted in contradictory results across some observations.

302 Once, it was reported that pinwheels are preferentially located around the centre of ocular

303 dominance domains ${ }^{28}$ where binocularity is relatively low. Because it was observed that the 
binocularity in the cortical neurons has positive correlation with the preferred spatial

305 frequency across the cortical surface ${ }^{9}$, pinwheels were expected to be located in regions

306 encoding low spatial frequency. However, in other, more precise optical imaging studies,

307 the orientation pinwheels are reported to be more probably located at either low or high

308 spatial frequency domains ${ }^{7,25}$ or in the positions between high- and low-frequency

309 regions ${ }^{43}$. Considering that estimating the pinwheel location can be affected by the

310 preprocessing condition found in the optical imaging methods (see Figure S2 in earlier

311 work $^{44}$ ), further studies imaging larger patches with higher resolution could provide a clue

312 to resolve this contradiction.

313 On the other hand, one might question why neural tuning is arranged randomly in

314 rodent V1, termed a salt-and-pepper map, and if our model can also address these issues in

315 rodents. Recent tracing studies revealed that feature preference may not be randomly

316 distributed in rodent V1, but is spatially clustered within a short distance that could not be

317 observed with older techniques ${ }^{45,46}$. Apart from the distinct retinal organization found in

318 higher mammals and rodents, another crucial factor in determining the scale of spatial

319 clustering may be convergent projection in the feedforward visual pathway ${ }^{39}$. Compared to

320 the localized convergence (almost one-to-one) of retinogeniculate connections in higher

321 mammals $^{47}$, the network of retinogeniculate connection is much more complex in rodents,

322 so that a single LGN neuron receives input from a number of different types of RGCs ${ }^{48}$.

323 Additional tracing experiments may reveal structural differences in the visual pathways of

324 rodents and higher mammals and explain the origin of the diversity of functional maps

325 across species.

The present scope of our model is limited to explaining how the retinal inputs could 327 provide the "original" blueprint for each functional map. Obviously, the refinement of the 
328 circuits from cortical activity and visual experience is also a critical factor modifying the

329 layout of functional maps ${ }^{23}$. To understand fully the development of the cortical circuits, it

330 must be further investigated which component of the cortical circuit activity, such as cross-

331 orientation suppression ${ }^{49}$, plays the most important role in each visual processing function.

332 Overall, our results suggest that the structure of retinal mosaics may provide an

333 initial framework for multiple functional maps. This common origin may enable the

334 development of various topographical correlations between different functional maps. 


\section{References}

1. LeVay, S., Connolly, M., Houde, J. \& Van Essen, D. The complete pattern of ocular dominance stripes in the striate cortex and visual field of the macaque monkey. $J$. Neurosci. 5, 486-501 (1985).

2. Blasdel, G. G. \& Salama, G. Voltage-sensitive dyes reveal a modular organization in monkey striate cortex. Nature 321, 579-585 (1986).

3. Shmuel, A. \& Grinvald, A. Functional organization for direction of motion and its relationship to orientation maps in cat area 18. J. Neurosci. 16, 6945-6964 (1996).

4. Movshon, J. A., Thompson, I. D. \& Tolhurst, D. J. Receptive field organization of complex cells in the cat's striate cortex. J. Physiol. 283, 79-99 (1978).

5. Horton, J. C. \& Adams, D. L. The cortical column: A structure without a function. Philos. Trans. R. Soc. Lond., B, Biol. Sci. 360, 837-862 (2005).

6. Van Hooser, S. D. Similarity and diversity in visual cortex: is there a unifying theory of cortical computation? Neurosci. 13, 639-656 (2007).

7. Hübener, M., Shoham, D., Grinvald, A. \& Bonhoeffer, T. Spatial relationships among three columnar systems in cat area 17. J. Neurosci. 17, 9270-9284 (1997).

8. Nauhaus, I., Nielsen, K. J., Disney, A. A. \& Callaway, E. M. Orthogonal microorganization of orientation and spatial frequency in primate primary visual cortex. Nat. Neurosci. 15, 1683-1690 (2012).

9. Nauhaus, I., Nielsen, K. J. \& Callaway, E. M. Efficient Receptive Field Tiling in Primate V1. Neuron 91, 893-904 (2016).

10. Swindale, N. V., Shoham, D., Grinvald, A., Bonhoeffer, T. \& Hübener, M. Visual cortex maps are optimized for uniform coverage. Nat. Neurosci. 3, 822-826 (2000).

11. Nauhaus, I. \& Nielsen, K. J. Building maps from maps in primary visual cortex. Curr. Opin. Neurobiol. 24, 1-6 (2014).

12. Chung, S. \& Ferster, D. Strength and orientation tuning of the thalamic input to simple cells revealed by electrically evoked cortical suppression. Neuron 20, 11771189 (1998).

13. Lien, A. D. \& Scanziani, M. Tuned thalamic excitation is amplified by visual cortical circuits. Nat. Neurosci. 16, 1315-1323 (2013).

14. Jin, J., Wang, Y., Swadlow, H. a \& Alonso, J. M. Population receptive fields of ON and OFF thalamic inputs to an orientation column in visual cortex. Nat. Neurosci. 14, 232-238 (2011).

15. Kremkow, J., Jin, J., Wang, Y. \& Alonso, J. M. Principles underlying sensory map topography in primary visual cortex. Nature 533, 52-57 (2016). 
16. Lee, K.-S., Huang, X. \& Fitzpatrick, D. Topology of ON and OFF inputs in visual cortex enables an invariant columnar architecture. Nature 533, 90-94 (2016).

17. Lien, A. D. \& Scanziani, M. Cortical direction selectivity emerges at convergence of thalamic synapses. Nature 558, 80-86 (2018).

18. Ringach, D. L. Haphazard Wiring of Simple Receptive Fields and Orientation Columns in Visual Cortex. J. Neurophysiol. 92, 468-476 (2004).

19. Ringach, D. L. On the origin of the functional architecture of the cortex. PLoS One 2, e251 (2007).

20. Soodak, R. E. The retinal ganglion cell mosaic defines orientation columns in striate cortex. Proc. Natl. Acad. Sci. U. S. A. 84, 3936-3940 (1987).

21. Paik, S. B. \& Ringach, D. L. Retinal origin of orientation maps in visual cortex. Nat. Neurosci. 14, 919-925 (2011).

22. Paik, S.-B. \& Ringach, D. L. Link between orientation and retinotopic maps in primary visual cortex. Proc. Natl. Acad. Sci. U. S. A. 109, 7091-7096 (2012).

23. Crair, M. C., Gillespie, D. C. \& Stryker, M. P. The role of visual experience in the development of columns in cat visual cortex. Science 279, 566-570 (1998).

24. Muir, D. R. et al. Embedding of cortical representations by the superficial patch system. Cereb. Cortex 21, 2244-2260 (2011).

25. Issa, N. P., Trepel, C. \& Stryker, M. P. Spatial Frequency Maps in Cat Visual Cortex. J. Neurosci. 20, 8504-8514 (2000).

26. Xu, X., Bosking, W. H., White, L. E., Fitzpatrick, D. \& Casagrande, V. A. Functional organization of visual cortex in the prosimian bush baby revealed by optical imaging of intrinsic signals. J. Neurophysiol. 94, 2748-2762 (2005).

27. Kisvarday, Z. F., Buzas, P. \& Eysel, U. T. Calculating Direction Maps from Intrinsic Signals revealed by Optical Imaging. Cereb. Cortex 11, 636-647 (2001).

28. Crair, M. C., Ruthazer, E. S., Gillespie, D. C. \& Stryker, M. P. Ocular dominance peaks at pinwheel center singularities of the orientation map in cat visual cortex. $J$. Neurophysiol. 77, 3381-3385 (1997).

29. Weliky, M., Bosking, W. H. \& Fitzpatrick, D. A systematic map of direction preference in primary visual cortex. Nature 379, 725-728 (1996).

30. Roerig, B. \& Kao, J. P. Organization of intracortical circuits in relation to direction preference maps in ferret visual cortex. J. Neurosci. 19, RC44 (1999).

31. Wassle, H., Boycott, B. B. \& Illing, R.-B. Morphology and Mosaic of on- and offBeta Cells in the Cat Retina and Some Functional Considerations. Proc. R. Soc. B Biol. Sci. 212, 177-195 (1981).

32. Chichilnisky, E. J. \& Kalmar, R. S. Functional asymmetries in ON and OFF ganglion cells of primate retina. J. Neurosci. 22, 2737-2747 (2002). 
408

409

410

411

412

413

414

415

416

417

418

419

420

421

422

423

424

425

426

427

428

429

430

431

432

433

434

435

436

437

438

439

440

441

442

443

444

445

33. Shatz, C. J. The prenatal development of the cat's retinogeniculate pathway. J. Neurosci. 3, 482-499 (1983).

34. Tusa, R. J., Palmer, L. A. \& Rosenquist, A. C. The retinotopic organization of area 17 (striate cortex) in the cat. J. Comp. Neurol. 177, 213-235 (1978).

35. Tootell, R. B., Switkes, E., Silverman, M. S. \& Hamilton, S. L. Functional anatomy of macaque striate cortex. II. Retinotopic organization. J. Neurosci. 8, 1531-1568 (1988).

36. Ohzawa, I., DeAngelis, G. C. \& Freeman, R. D. Encoding of binocular disparity by simple cells in the cat's visual cortex. J. Neurophysiol. 75, 1779-1805 (1996).

37. Löwel, S. et al. The layout of orientation and ocular dominance domains in area 17 of strabismic cats. Eur. J. Neurosci. 10, 2629-2643 (1998).

38. Landisman, C. E. \& Ts'o, D. Y. Color processing in macaque striate cortex: relationships to ocular dominance, cytochrome oxidase, and orientation. $J$. Neurophysiol. 87, 3126-3137 (2002).

39. Jang, J. \& Paik, S.-B. Interlayer Repulsion of Retinal Ganglion Cell Mosaics Regulates Spatial Organization of Functional Maps in the Visual Cortex. $J$. Neurosci. 37, 12141-12152 (2017).

40. Levay, S., Stryker, M. P. \& Shatz, C. J. Ocular dominance columns and their development in layer IV of the cat's visual cortex: A quantitative study. J. Comp. Neurol. 179, 223-244 (1978).

41. Crair, M. C., Ruthazer, E. S., Gillespie, D. C. \& Stryker, M. P. Relationship between the Ocular Dominance and Orientation Maps in Visual Cortex of Monocularly Deprived Cats. Neuron 19, 307-318 (1997).

42. Derrington, A. M. Development of spatial frequency selectivity in striate cortex of vision-deprived cats. Exp. Brain Res. 55, 431-437 (1984).

43. Ribot, J., Romagnoni, A., Milleret, C., Bennequin, D. \& Touboul, J. Pinwheel-dipole configuration in cat early visual cortex. Neuroimage 128, 63-73 (2016).

44. Kaschube, M. et al. Universality in the evolution of orientation columns in the visual cortex. Science 330, 1113-1116 (2010).

45. Ringach, D. L. et al. Spatial clustering of tuning in mouse primary visual cortex. Nat. Commun. 7, 12270 (2016).

46. Kondo, S., Yoshida, T. \& Ohki, K. Mixed functional microarchitectures for orientation selectivity in the mouse primary visual cortex. Nat. Commun. 7, 13210 (2016).

47. Usrey, W. M., Reppas, J. B. \& Reid, R. C. Specificity and strength of retinogeniculate connections. J. Neurophysiol. 82, 3527-3540 (1999).

48. Morgan, J. L., Berger, D. R., Wetzel, A. W. \& Lichtman, J. W. The Fuzzy Logic of Network Connectivity in Mouse Visual Thalamus. Cell 165, 192-206 (2016). 
49. Koch, E., Jin, J., Alonso, J. M. \& Zaidi, Q. Functional implications of orientation maps in primary visual cortex. Nat. Commun. 7, 13529 (2016).

50. Gauthier, J. L. et al. Receptive fields in primate retina are coordinated to sample visual space more uniformly. PLoS Biol. 7, e1000063 (2009).

51. Nauhaus, I., Benucci, A., Carandini, M. \& Ringach, D. L. Neuronal Selectivity and Local Map Structure in Visual Cortex. Neuron 57, 673-679 (2008).

452

52. Obermayer, K. \& Blasdel, G. G. Geometry of orientation and ocular dominance columns in monkey striate cortex. J. Neurosci. 13, 4114-4129 (1993).

53. Croner, L. J. \& Kaplan, E. Receptive fields of P and M ganglion cells across the primate retina. Vision Res. 35, 7-24 (1995).

54. Balasubramanian, V.\& Sterling, P. Receptive fields and functional architecture in the retina. J. Physiol. 587, 2753-2767 (2009).

55. Sejnowski, T. J. Storing covariance with nonlinearly interacting neurons. J. Math. Biol. 4, 303-321 (1977).

56. Gauthier, J. L. et al. Uniform signal redundancy of parasol and midget ganglion cells in primate retina. J. Neurosci. 29, 4675-4680 (2009). 
464

465

466

467

468

469

470

471

472

\section{Methods}

The simulations were performed based on the statistical wiring model ${ }^{18,19}$. Here we briefly summarize the algorithm and the parameters used in the simulations.

\section{Structure of retinal ganglion cell mosaics}

To simulate the development of binocular afferents to the V1, the contra- and ipsilateral RGC mosaics were individually generated by the superposition of ON and OFF RGC mosaics. The mosaic of each type of RGC was generated by adding random displacement to each vertex of a hexagonal lattice that represents the position of receptive fields. The centres of RGC receptive field position vectors are defined by

$$
\begin{aligned}
& \boldsymbol{p}_{i j, \text { cont }}^{O F F}=d \boldsymbol{L}_{i j}^{R G C}+\boldsymbol{\eta}_{i j} \\
& \boldsymbol{p}_{i j, \text { cont }}^{O N}=(1+\alpha) d R_{\theta} \boldsymbol{L}_{i j}^{R G C}+\boldsymbol{\eta}_{i j}+\boldsymbol{\eta}_{0} \\
& \boldsymbol{p}_{i j, i p s i}^{O F F}=d R_{\phi, i p s i} \boldsymbol{L}_{i j}^{R G C}+\boldsymbol{\eta}_{i j} \\
& \boldsymbol{p}_{i j, i p s i}^{O N}=(1+\alpha) d R_{\phi, i p s i} R_{\theta} \boldsymbol{L}_{i j}^{R G C}+\boldsymbol{\eta}_{i j}+\boldsymbol{\eta}_{0} \\
& \boldsymbol{L}_{i j}^{R G C}=\frac{1}{2}\left[\begin{array}{cc}
1 & 1 \\
\sqrt{3} & -\sqrt{3}
\end{array}\right]\left[\begin{array}{c}
i \\
j
\end{array}\right] i, j=0, \pm 1, \pm 2, \cdots
\end{aligned}
$$

Here, $\boldsymbol{L}_{i j}^{R G C}$ is the vertices of a unit hexagonal grid where $d$ represents the grid spacing for the OFF mosaic, and $(1+\alpha) d$ represents the grid spacing for the ON mosaic.

The value of $d, \alpha$ was collected from published reconstruction data of RGC receptive fields of $M$. fascicularis ${ }^{50}$. The $\boldsymbol{\eta}_{i j}$, a random positional noise of RGC mosaics, is modelled as two-dimensional Gaussian noise to have a zero mean value with standard deviation $\sigma_{n}$. To generate realistic RGC mosaics, $\sigma_{n}$ was set to match the conditions of mean nearestneighbour distance and noisiness observed in macaque monkey retinal mosaics ${ }^{34,50}$. A random relative spatial shift between ON and OFF mosaics $\boldsymbol{\eta}_{0}$ was also added. 
The matrix

$$
R_{\theta}=\left[\begin{array}{cc}
\cos \theta & \sin \theta \\
-\sin \theta & \cos \theta
\end{array}\right]
$$

represents the relative rotation between the $\mathrm{ON}$ and OFF mosaics where $\theta$ is set to satisfy a scale factor proper for regenerating the actual period of orientation maps observed in macaque monkeys ${ }^{51,52}$. The matrix

$$
R_{\phi}=\left[\begin{array}{cc}
\cos \phi & \sin \phi \\
-\sin \phi & \cos \phi
\end{array}\right]
$$

represents relative rotation between contralateral and ipsilateral RGC mosaics, where $\phi$ is a random value between 0 and $\pi$. All the parameter details are shown in Table 1 .

Initial conditions for connectivity parameters and receptive field models model of 2D Gaussian and their linear sum, respectively. The standard deviation of the

504 surrounding region was set to three times that of the centre region ${ }^{53}$.

At the initial stage of development, we assumed that the RGCs are statistically wired to cortical space with two-dimensional Gaussian function with a standard deviation of $\sigma_{c o n}$.

$$
w_{i j}=\frac{W_{\text {init }}}{\sigma_{\text {con }} \sqrt{2 \pi}} \exp \left(-\frac{\left(d_{i j}+\boldsymbol{\eta}_{V 1, j}\right)^{2}}{2 \sigma_{c o n}^{2}}\right)
$$

Here, $w_{i}$ represents the synaptic weighting between $\mathrm{i}^{\text {th }} \mathrm{RGC}$ and $\mathrm{j}^{\text {th }}$ cortical sites, where $W_{\text {init }}$ is the initial connection weight. For realistic retinotopic projection, we added zero mean two-dimensional Gaussian noise, $\boldsymbol{\eta}_{V 1}$, with a standard deviation of $\sigma_{R}$.

The receptive fields of RGCs and V1 neurons were defined as a centre-surround 


$$
\begin{gathered}
\Psi_{i, R G C}= \pm\left(\frac{1}{\sigma \sqrt{2 \pi}} \exp \left(-\frac{r_{i}^{2}}{2 \sigma_{R G C}^{2}}\right)-\frac{1}{3 \sigma \sqrt{2 \pi}} \exp \left(-\frac{r_{i}^{2}}{18 \sigma_{R G C}^{2}}\right)\right)(+: \text { ON cell, }-: \text { OFF cell }) \\
\Psi_{j, V 1}=\sum_{i} w_{i j} \cdot \Psi_{i}^{R G C}
\end{gathered}
$$

$508 \boldsymbol{r}_{i}$ is a distance vector from the centre of $i^{\text {th }} \mathrm{RGC}$ to each position of the visual field. In this

509 case, $\sigma_{O N, R G C}$ and $\sigma_{O F F, R G C}$ were set as $(1+\alpha) d / 2$ and $d / 2$ to satisfy the condition that the receptive fields of ON and OFF RGC mosaics cover all of the visual field ${ }^{54}$.

\section{Nonlinear response curve of a single neuron}

513 The response curves of RGCs and V1 neurons were designed based on the linear-nonlinear

514 model, which shows a normalized response to visual input $\boldsymbol{S}$ through a sigmoidal kernel

$$
\begin{aligned}
& R_{i, R G C}(\boldsymbol{S})=\left(1+\exp \left(-\frac{\Psi_{i, R G C} \cdot \boldsymbol{S}}{\delta_{R G C}}\right)\right)^{-1} \\
& R_{j, V 1}(\boldsymbol{S})=\left(1+\exp \left(-\frac{I-0.5}{\delta_{V 1}}\right)\right)^{-1} \\
& I=\sum_{i} w_{i j} \cdot R_{i, R G C}
\end{aligned}
$$

518 where $R_{i, R G C}(\boldsymbol{S})$ and $R_{j, V 1}(\boldsymbol{S})$ are the response of $\mathrm{i}^{\text {th }} \mathrm{RGC}$ and $\mathrm{j}^{\text {th }}$ cortical cell for visual

519 stimuli $S$. Here, $\delta$ stands for nonlinearity of the sigmoidal response function. All the 520 parameter details are shown in Table 1. 
523 In our binocular development model, the development of connections between contralateral

524 RGCs and V1 neurons were first simulated, and then the ipsilateral connections were

525 allowed to develop. During development, drifting grating was given to binocular RGC

526 mosaics where the drifting grating was designed as a sine function of twenty different

527 spatial frequencies $(0.5-8$ cycles/degree $)$ and twenty orientations $(0-\pi)$. The synaptic

528 weights were updated following a simple covariance rule ${ }^{55}$ as follows

531 where the learning threshold $\bar{R}$ is defined as the average value of the current response of the 532 cortical cell. The $\tau$ represents a time constant showing how rapidly the threshold changes.

533 The $\epsilon$ represents the learning rate, how fast the synaptic weights are updated. Note that we 534 assumed that there is a limitation of resource, so both $w_{i j}$ and $\sum_{i} w_{i j}$ have upper limit $w_{l i m}$ 535 and $W_{\text {lim }}$. The development of both contra- and ipsilateral pathways were simulated with 536 drifting grating inputs for 10,000 frames.

\section{Measurement of cortical functional maps}

539 At the initial stage of development, we assumed that the RGCs are statistically wired to 540 cortical space in a two-dimensional Gaussian function with a standard deviation of $\boldsymbol{\sigma}_{\boldsymbol{c o n}}$.

$$
\theta_{O P}=\frac{\arg \left(\int|\Psi(\omega) \| \omega| \exp (2 \operatorname{iarg}(\omega)) d \omega\right)}{2}
$$

$$
O S I=\frac{\int\left|\Psi\left(\omega_{\text {pref }}, \theta\right)\right| \exp (2 i \theta) d \theta}{\int\left|\Psi\left(\omega_{\text {pref }}, \theta\right)\right| d \theta}
$$

$$
\omega_{\text {pref }}=\left|\int\right| \Psi(\omega)|| \omega|\exp (2 \operatorname{iarg}(\omega)) d \omega|
$$



between the centre positions of ON and OFF RGCs.

$$
\theta_{D P, k}=\arg \left(\frac{\sum_{i} w_{i k} \cdot \boldsymbol{r}_{i}^{O F F}}{\sum_{i} w_{i k}}-\frac{\sum_{j} w_{j k} \cdot \boldsymbol{r}_{j}^{O N}}{\sum_{j} w_{j k}}\right)
$$

The ocular dominance was calculated as the relative strength of the mean cortical response $\overline{R_{V 1}}$, to visual stimulus $S$, given to contra- and ipsilateral RGCs.

$$
O D=\frac{\overline{R_{V 1}\left(S_{\text {contra }}\right)}-\overline{R_{V 1}\left(S_{\text {lpsl }}\right)}}{\overline{R_{V 1}\left(S_{\text {contra }}\right)}+\overline{R_{V 1}\left(S_{\text {lpsl }}\right)}}
$$

To measure the spatial frequency, we computed the response of cortical cells to

551 drifting gratings of various orientation and spatial frequency. The preferred spatial

552 frequency was defined as the value of spatial frequency that induces the maximum

553 response. To implement a realistic map-topography, each map was simulated from the

554 hexagonal RGC mosaics with a realistic level of spatial noise in the cell position ${ }^{21}$ and all

555 the simulated maps in the study (except in Figure $2 \mathrm{~g}-\mathrm{h}$ ) were averaged from 20 repeated 556 trials of simulation.

\section{Analysis of cortical functional maps}

559 To measure the intersection angle between map gradients, each functional map was first

560 smoothed using a 2D Gaussian filter $(\sigma=d / 5)$. The gradients of OD and SF maps were

561 calculated from the differences between the corresponding pixels of each map. The

562 gradients of OP and DP maps were calculated in the complex domain: $C(O P)=\exp (2 i *$

$563 O P)$ and $C(D P)=\exp (i * D P)$. The intersection angle between maps was properly scaled 564 into the $[0, \pi]$ domain. 

changes at a given site, and was defined as the sum of complex vectors of OP or DP. In this

\section{7}

The overlap ratio between direction flip area and iso-orientation area was calculated as the ratio of overlapped regions to the entire area of iso-orientation domains. A direction flip was defined as the regions having the bottom 5\% of LHIDP and iso-orientation domain was defined as the regions having the top $5 \%$ of LHIop.

$$
\begin{aligned}
L H I_{O P} & =\left|\sum \frac{1}{\sigma_{L H I} \sqrt{2 \pi}} \exp \left(-\frac{\text { distance }^{2}}{2 \sigma_{L H I}^{2}}\right) \exp (2 i * O P)\right| \\
L H I_{D P} & =\left|\sum \frac{1}{\sigma_{L H I} \sqrt{2 \pi}} \exp \left(-\frac{\text { distance }^{2}}{2 \sigma_{L H I}^{2}}\right) \exp (i * D P)\right|
\end{aligned}
$$
calculation, a 2D Gaussian weighting of cortical distance was applied ${ }^{49}$.

\section{Experimental data of functional maps and multi-electrode recordings}

Multi-electrode data recorded from cats were provided by Jose-Manuel Alonso (ref. 15 and personal communication). The functional maps in the cat are from ref. 7, 10, 25, 27, 28 and 37 (orientation map), ref. 27 (direction map), ref. 7, 10, 25, 28 and 37 (ocular dominance map), and ref. 7, 10 and 25 (spatial frequency map). The functional maps in the monkey are from ref. 8, 9 and 38 (orientation map), ref. 9 and 38 (ocular dominance map) and ref. 8 and 9 (spatial frequency map). Orientation and ocular dominance maps in the galago are from 


\section{Acknowledgments}

585 We are grateful to Jose-Manuel Alonso (State University of New York) for sharing

586 receptive field data on the cat primary visual cortex. This research was supported by the

587 Basic Science Research Program through the National Research Foundation of Korea

588 (NRF), funded by the Ministry of Science and ICT (NRF-2016R1C1B2016039, NRF-

589 2016R1E1A2A01939949) (to S.P.).

591 Author contributions

592 S.P. conceived the study. M.S., J.J. and S.P. designed the model. M.S. and J.J. performed

593 the simulations. M.S., J.J., G.K. and S.P. analysed the data. M.S., J.J. and S.P. wrote the

594 manuscript. All authors discussed and commented on the manuscript.

597 The authors declare that they have no competing interests. 
Table 1

\section{Parameters}

Nearest OFF RGC distance

ON-OFF scaling factor

Standard deviation of RGC positional noise

Standard deviation of RGC to Cortical connection

Standard deviation of retinotopic noise

Nonlinearity of sigmoidal kernel

Nonlinearity of sigmoidal kernel

Resource limit of single connection

Resource limit of total connection

Time constant of firing rate average

Standard deviation of Gaussian kernel used in LHI calculation

Learning rate in Hebbian learning

\section{Letter}

$d$

$\alpha$

$\sigma_{n}$

$\sigma_{\text {con }}$

$60 \mu m$

$\sigma_{R}$

$71 \mu m$

$\delta_{R G C}$

0.1

$\delta_{V 1}$

0.15

$w_{\text {lim }}$

0.15

$W_{\text {lim }}$

1

$\tau$

10 frame

$\sigma_{L H I}$ $28 \mu m$

$\epsilon$ 
a

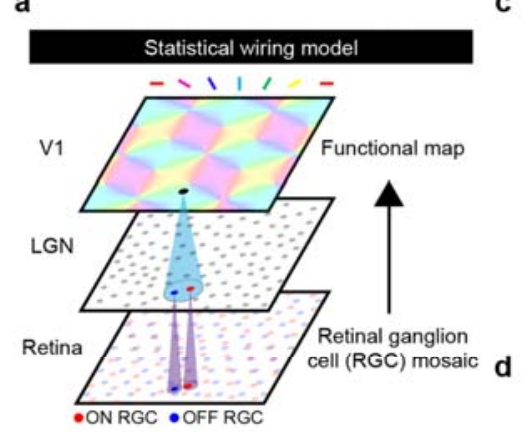

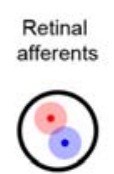

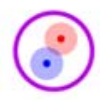

Retinal ON/OFF afferents response
600

601

603

604

605

606

607
() $\odot \mathbf{c}$ Ce

○

b

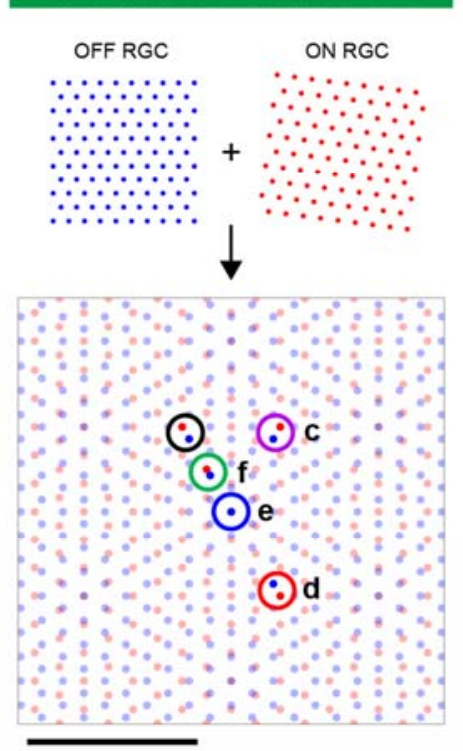

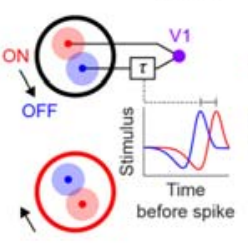

e Contralateral
retinal afferents<smiles>c1ccc2ccccc2c1</smiles>
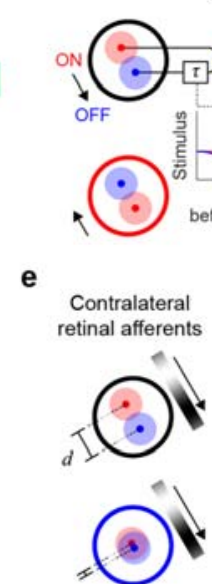

f
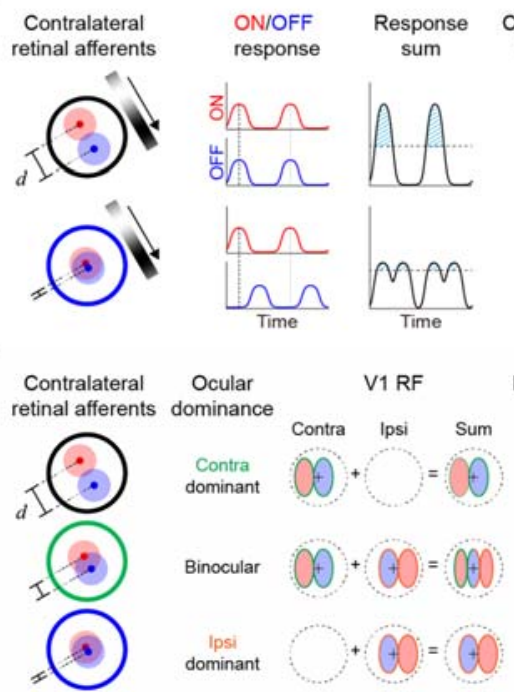
ON/OFF
response

Response
sum
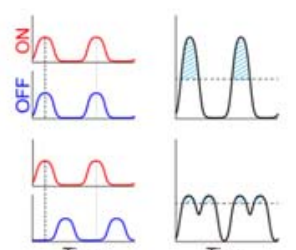

g
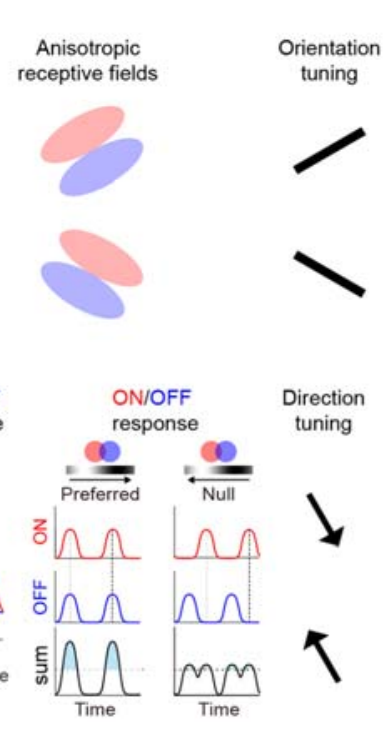

Contralatera response
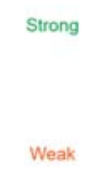

requency

tuning

Low

High

Low
Orientation preference

(OP)

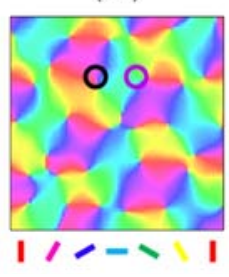

Direction preference (DP)

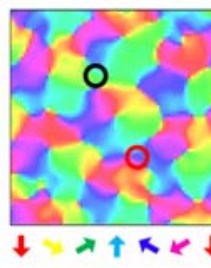

Ocular dominance

(OD)

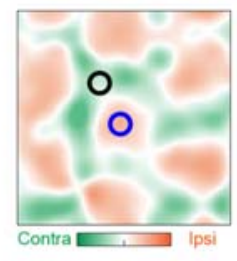

Spatial frequency (SF)

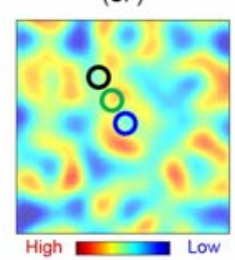

\section{Figure 1. Retinal origin of various functional maps in V1}

a, Developmental model of cortical functional maps as a feedforward projection of ON and OFF retinal ganglion cell (RGC) mosaics. $\mathbf{b}$, The moiré interference pattern between ON and OFF RGC mosaics in the contralateral retina provides a common framework of hexagonal patterns for cortical maps. The scale bar indicates the period of the interference pattern. c, With the local feedforward pooling of RGCs, orientation tuning of $\mathrm{V} 1$ neurons is induced from the alignment of 
608 ON and OFF subregions. d, Temporal delay between ON and OFF pathways result 609 in a direction-selective response of V1 neurons. Preferred direction of the neuron is

610 from ON to OFF centres of RGCs. e, According to the distance between

611 neighbouring RGCs in the contralateral retina, the degree of overlap between ON

612 and OFF subregions in V1 receptive fields varies and differential response occurs.

613 When ON and Off subregions are far from each other, the V1 neuron can generate

614 a strong response to contralateral input. If ON and OFF RGCs are very close; they

615 are not simultaneously activated and cannot respond strongly to contralateral

616 inputs. f, In binocular regions, phase difference between the contra- and ipsilateral

617 receptive field induces preference for higher spatial frequency. $\mathbf{g}$, The common

618 retinal origin of each functional map causes a topographical correlation among the

619 maps. The scale bar indicates the period of the orientation map, which is set up by

620 the interference pattern in $\mathbf{b}$. 


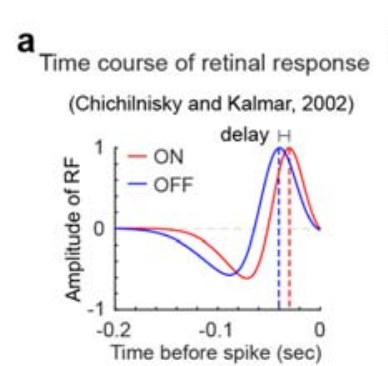

f

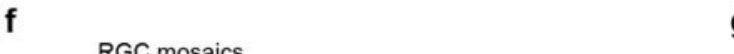

\section{b}

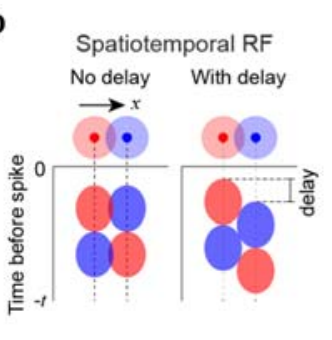

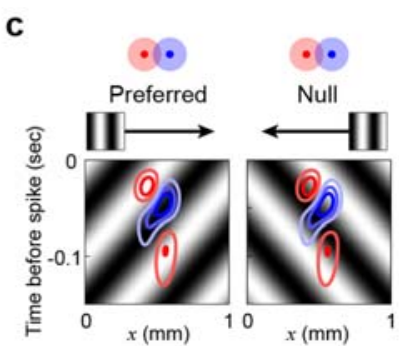
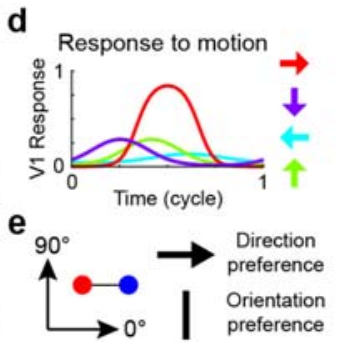

g

h

Orientation map with iso-domain

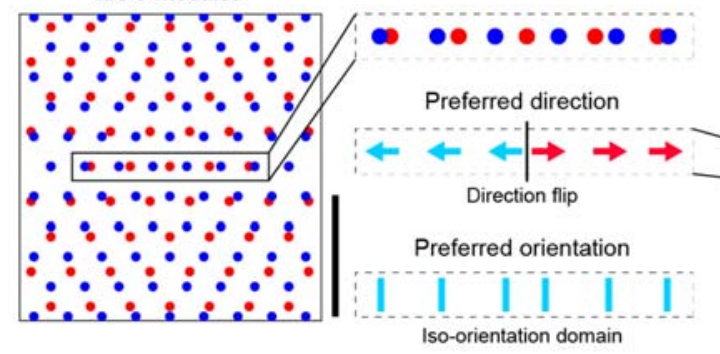

i

j

Direction flip and iso-orientation domain

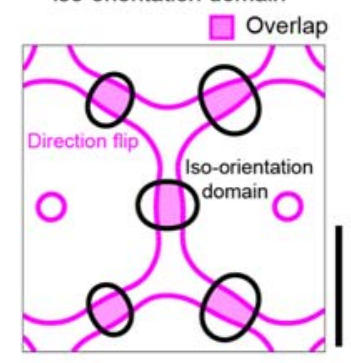

Overlap ratio

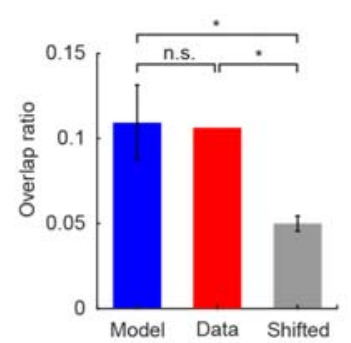

621

Figure 2. Flip of preferred direction at the centre of iso-orientation domains

a-e, Direction tuning of a V1 neuron originates from a temporal delay between ON and OFF pathways. a, Temporal dynamics of receptive fields indicates a consistent delay ( $~ 9 \mathrm{~ms}$ ) between ON and OFF pathways ${ }^{32}$. b. The ON-OFF delay induces a non-separable spatiotemporal receptive field of V1 neuron. c, This non-separable receptive field induces direction tuning with preferred response to ON-to-OFF direction. d, Direction tuning of a model V1 neuron. e, Preferred direction and orientation from an ON-OFF dipole structure. f, In moiré interference, the direction of ON-to-OFF RGC dipoles suddenly flips in areas where their orientation is 
631 consistent. The scale bar indicates the half period of the interference pattern. $\mathbf{g}-\mathbf{i}$,

632 Direction and orientation maps seeded from a common origin of the RGC mosaics

633 in $\mathbf{f}$. The scale bar indicates a half-of-map period. $\mathbf{g}$, Linear flips (white solid lines)

634 in a direction selectivity map. $\mathbf{h}$, Iso-orientation domains (black solid lines) in an

635 orientation map. $\mathbf{i}$, Direction flips in $\mathbf{g}$ and iso-orientation domains in $\mathbf{h}$. Direction flip

636 (pink solid lines) appears at the centre of the iso-orientation domains (black solid

637 lines). j, Both in model $(N=100)$ and animal data $(N=1)^{27}$, the overlap between

638 the two regions is significant, but not in randomly shifted data $(N=100)$. (model vs.

639 shifted: two-tailed t-test, $P<10^{-15}, T=-25.723$; data vs. shifted: one sample two-

640 tailed t-test, $P<10^{-15}, T=-126.146$; model vs. data: one sample two-tailed t-test,

641 n.s., $P=0.177, T=1.36$ ) Error bars indicate SD. $\mathbf{k}$, The average of overlap ratio in

642 the radial direction shows a local maximum at the origin and gradually decreases

643 as one map is spatially shifted, in both model and data ${ }^{27}$. Shaded region indicates

644 SD. 


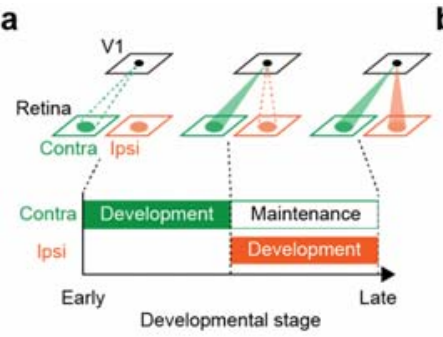

b

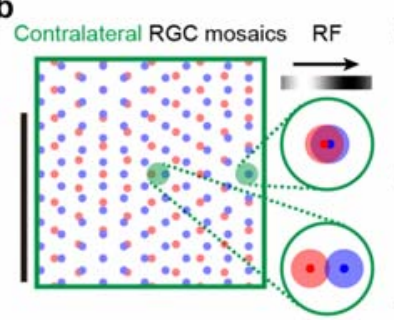

d

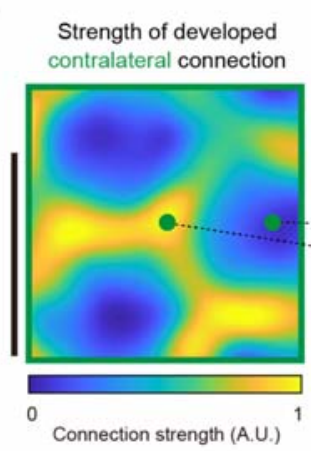

g

Multielectrode recording across V1
(Kremkow et al., 2016)
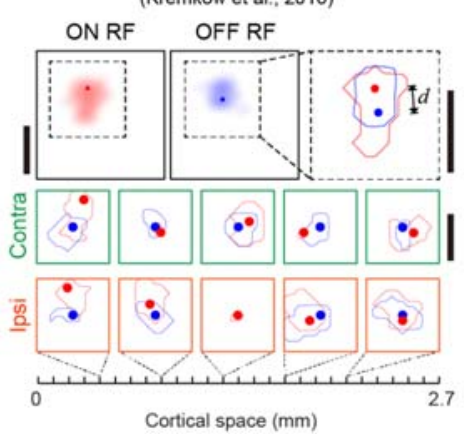

h

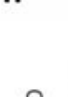

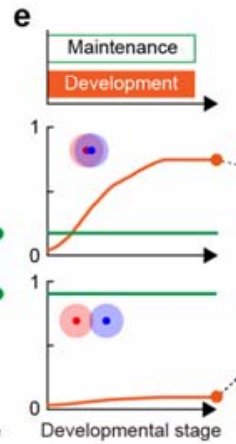
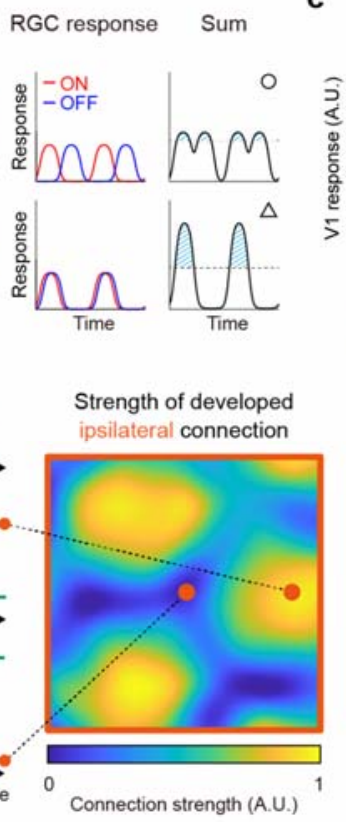

Connection strength (A.U.)

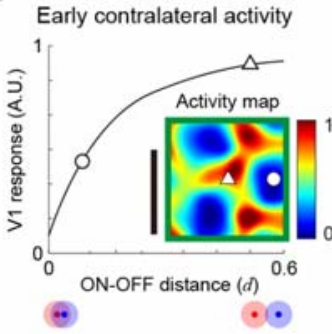

f

Ocular dominance (OD)

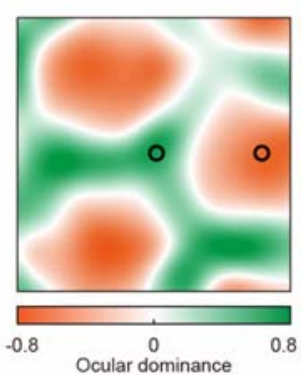

Auto-correlation

$-\mathrm{OD}-d_{\text {Contan }}-d_{\text {tosi }}$

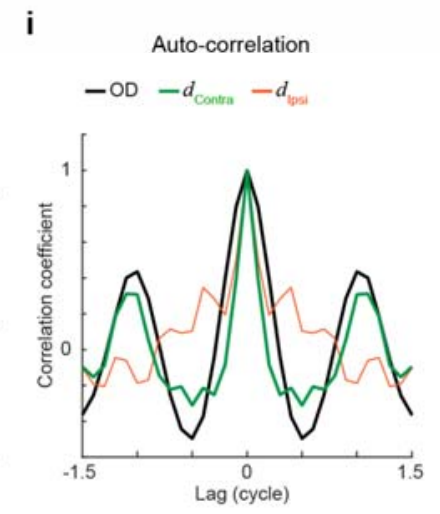

Periodic variation of $\mathrm{OD}$ and $d_{\text {contra }}$
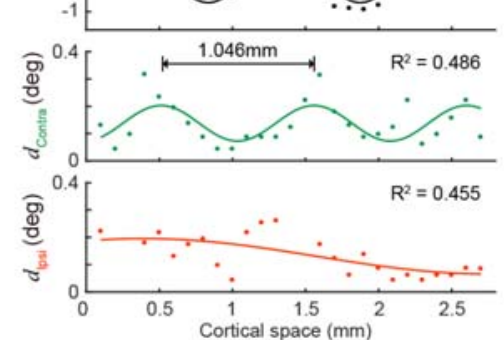

Figure 3. Ocular dominance map from the contralateral retinal mosaics

a, Sequential development model of contra- and ipsilateral visual pathways and

b, Distance between the neighbouring ON and OFF RGCs determines the degree of overlap in V1 receptive field subregions, and the cortical response. (Top) When two subregions greatly overlap, the neuron induces a weak

651 contralateral response. (Bottom) Where the two subregions show less overlap, the

652 V1 neuron generates stronger response. The scale bar indicates the period of the

653 interference pattern. c, The simulated cortical response is an increasing function of 
654 the ON-OFF RGC distance. Inset, an early periodic pattern of the cortical response

655 generated by contralateral RGC mosaics. d, (Left) Early periodic pattern of the

656 cortical response in c induces a periodic pattern of contralateral dominance. (Right)

657 Refinement of the contralateral feedforward connections. An activity-dependent

658 process strengthens feedforward connection when postsynaptic V1 neurons

659 strongly respond. The scale bar indicates a map period. e, Refinement of the

660 ipsilateral connections. Strength of total connections from contra- and ipsilateral

661 afferents to a V1 neuron is assumed to be a constant. f, Ocular dominance map

662 originated by $\mathbf{d}$ and e. g, Previous observation ${ }^{28}$ of receptive field and ocular

663 dominance recorded across the V1 using multiple electrodes. The scale bar

664 indicates $0.5^{\circ}$ in visual space. $\mathbf{h}$, Both ocular dominance and contralateral ON-OFF

665 distance appeared to vary periodically with the same spatial period ( 1 mm), and

666 were fitted to a sinusoidal curve (OD : $R^{2}=0.749$, contralateral: $R^{2}=0.486$,

667 ipsilateral: $\left.R^{2}=0.455\right)$. i, Auto-correlation of ocular dominance and ON-OFF

668 distance. 
a

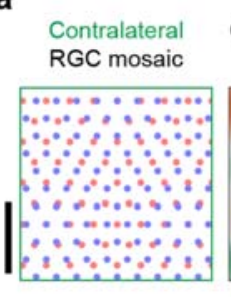

d

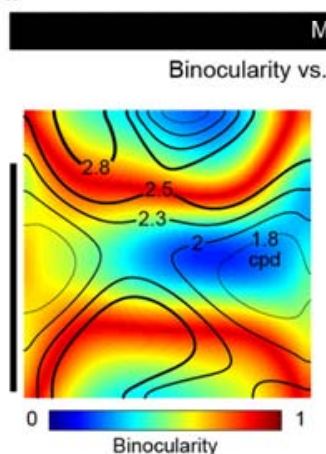

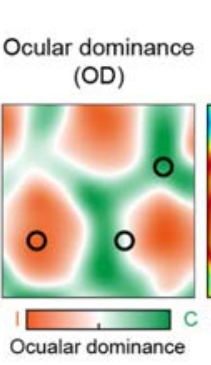

Ocualar dominance

\section{Model}

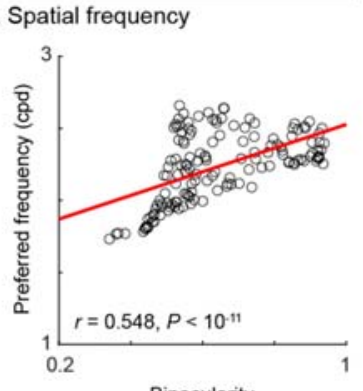

b

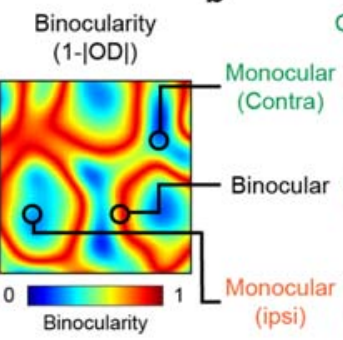

e
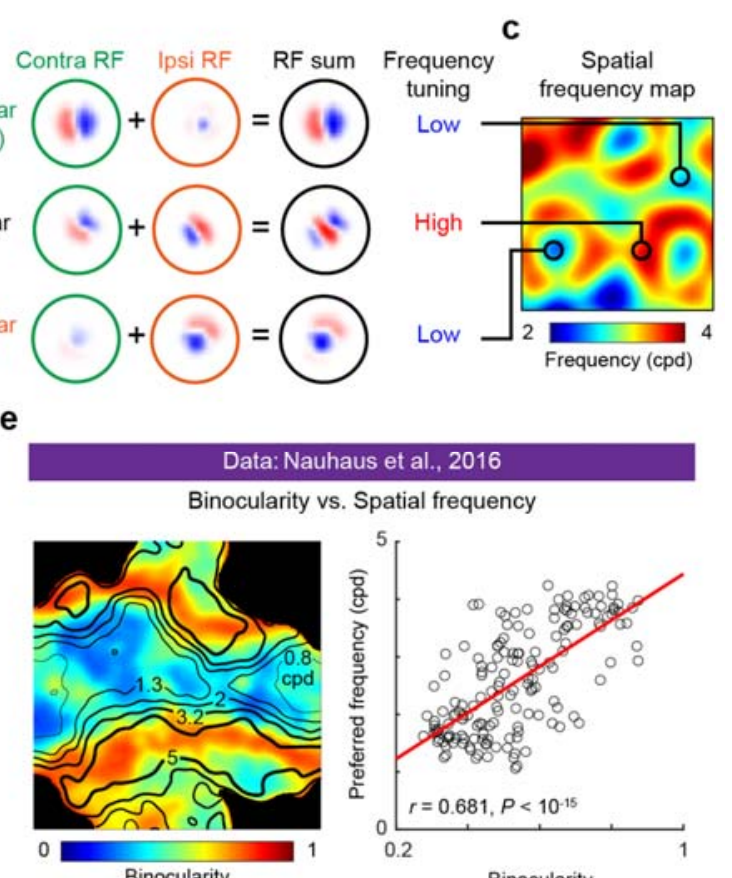

Figure 4. Spatial frequency map arises from the matching of contra- and ipsilateral afferents

a, Model ocular dominance map and its spatial organization of binocularity. $\mathbf{b}$, Intermingling of contra- and ipsilateral receptive fields in monocular and binocular regions. Phase difference between contra- and ipsilateral receptive fields in the binocular region induces narrower subregions of receptive field, resulting in tuning for higher spatial frequency. c, A model spatial frequency map induced from the RGC mosaics in a. d-e, Simulated (d) and observed ${ }^{9}$ (e) binocularity vs. spatial frequency maps. (Left) Binocularity map with contours of spatial frequency maps.

Thick contours indicate regions encoding high spatial frequency. (Right) A strong correlation between binocularity and spatial frequency maps was observed both in the model and data (Pearson correlation coefficient, model: $N=144, r=0.548, P=$ $1.082 \times 10^{-12}$, data: $\left.N=164, r=0.681, P<10^{-15}\right)$. The scale bar indicates a half period of the interference pattern or map. 
a

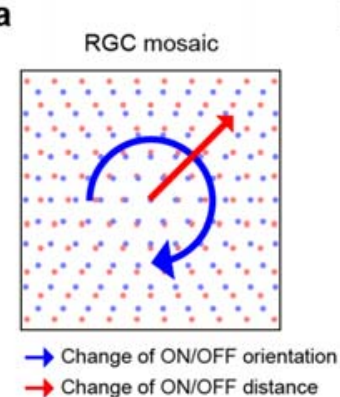

C

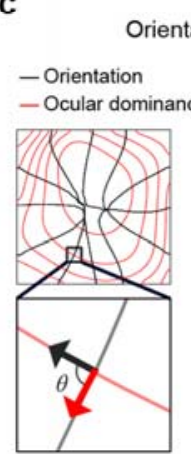

e

RGC mosaics

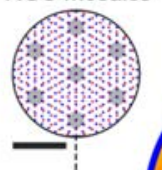

a, Spatial organization of ON-OFF RGC dipole distance and angular alignment in b

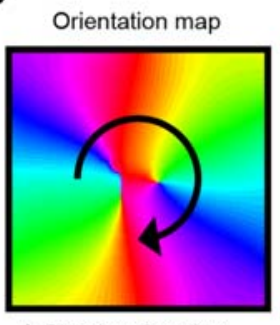

d
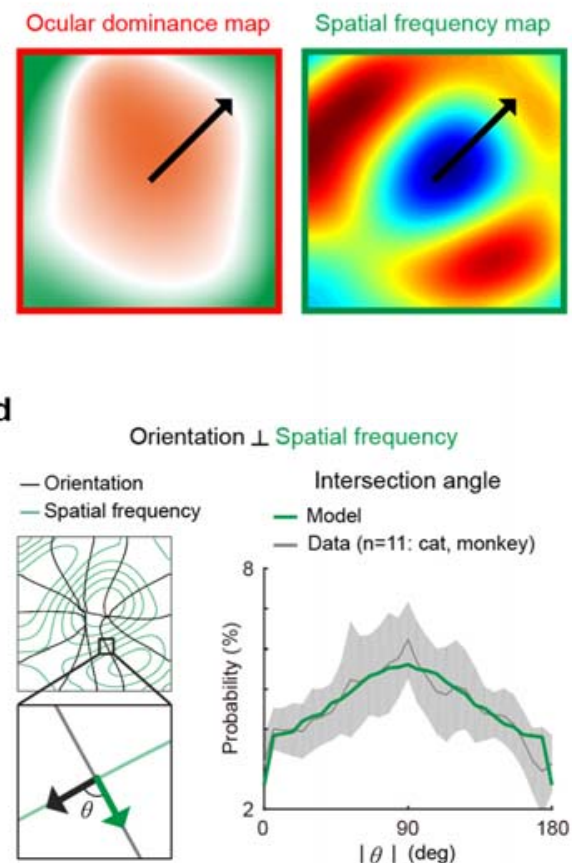

Orientation $\perp$ Spatial frequency
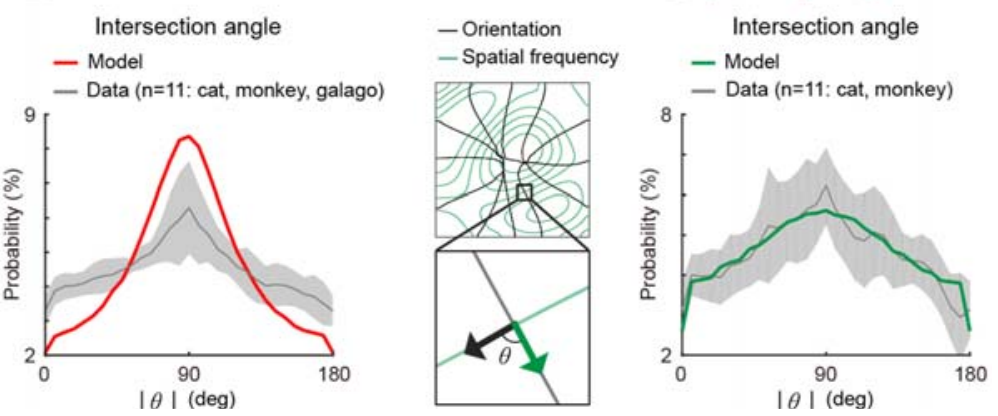

Spatial frequency map ( $\mathrm{n}=2: 2$ cat) ( $n=3: 1$ cat, 2 ferret)

Ocular dominancep map

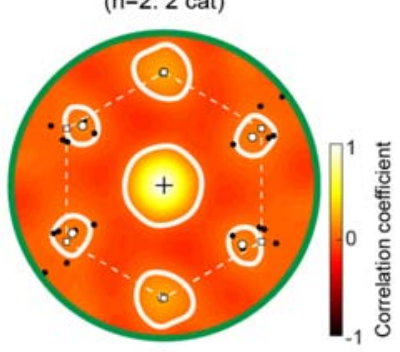

Figure 5. Topographical relationship and hexagonal periodicity of functional maps the moiré interference pattern. Note that the gradient of the two variables is orthogonal across the mosaics area. $\mathbf{b}$, Orientation preference changes in a circular direction around singularities, while ocular dominance and spatial frequency tunings change in a radial direction. c, Orthogonal distribution of intersection angle between the gradients of orientation and ocular dominance 
693 maps (11 pairs of maps; 6 cats, 4 monkeys, 1 galago). d, Orthogonal distribution of 694 intersection angle between the gradients of orientation and spatial frequency maps 695 (11 pairs of maps; 5 cats, 6 monkeys). e, The average autocorrelation patterns of 696 various functional maps from different species ${ }^{7,10,25-30}$. Peaks (open white circles)

697 in the autocorrelation closely matched the ones in a perfect hexagonal lattice (open

698 white squares). Solid black circles indicate peaks in the autocorrelation of

699 individual maps. The scale bar indicates a period of the interference pattern or 700 map. 
701 Supplementary material

\section{Universality of the developmental origins of diverse 703 functional maps in the visual cortex}

704 Min Song ${ }^{1,2 \dagger}$, Jaeson Jang ${ }^{1 \dagger}$, Gwangsu Kim $^{3}$ \& Se-Bum Paik ${ }^{1,2 *}$

$705{ }^{1}$ Department of Bio and Brain Engineering, ${ }^{2}$ Program of Brain and Cognitive Engineering,

$706{ }^{3}$ Department of Physics, Korea Advanced Institute of Science and Technology, Daejeon

707 34141, Republic of Korea

708

709

710 This PDF file includes:

$711 \quad$ Supplementary Figure 1 to 3 
a

Development of contralateral/ipsilateral connection

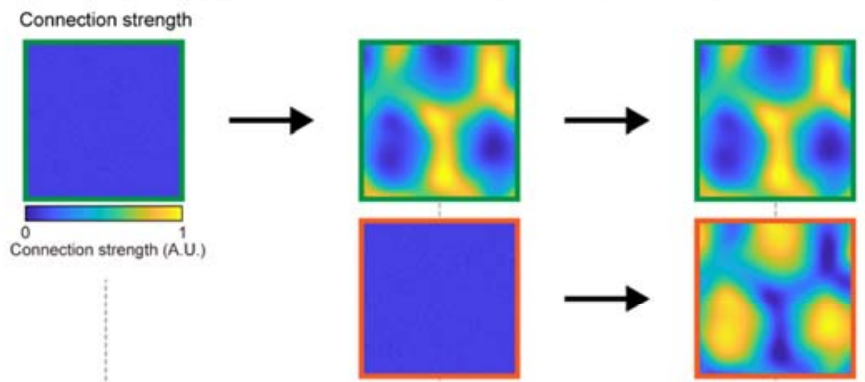

b

Binocular matching of orientation preferrence during development
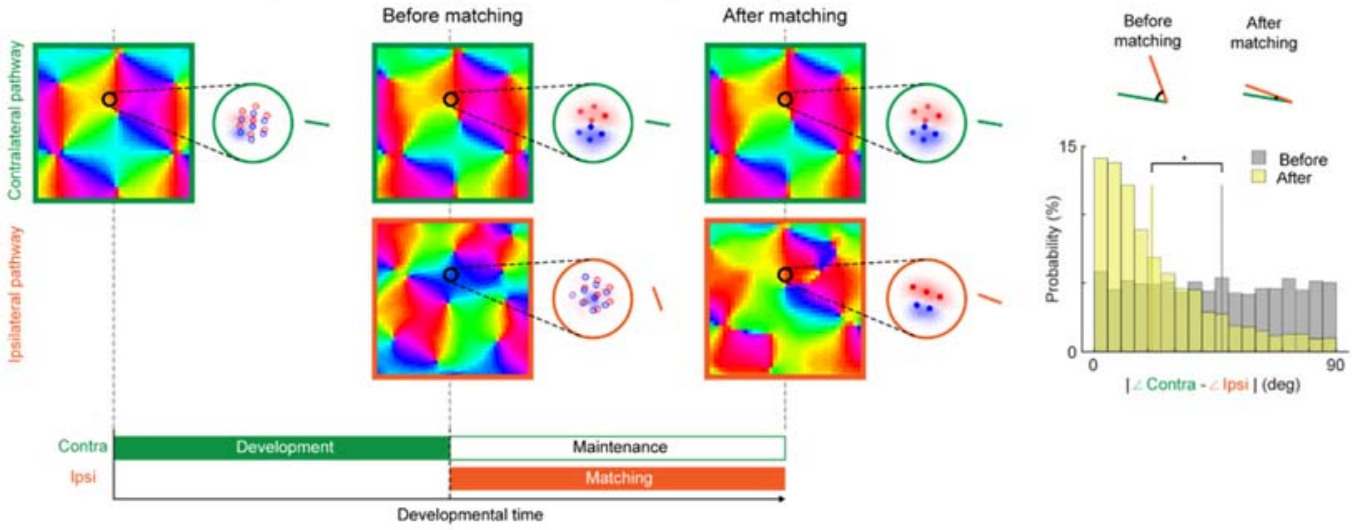

Supplementary Figure 1. Orientation matching between contra- and ipsilateral pathways

716 a, Change in the strength of connections from contra- and ipsilateral pathways to

717 V1 during development. (Left) Initially, only contralateral connections are weakly

718 connected to the V1. (Middle) The contralateral connections are selectively

719 strengthened by activity of postsynaptic V1 neurons. (Right) The ipsilateral

720 connections are set up differentially, according to the connection strength from the

721 contralateral pathway. b, Matching process of receptive fields and orientation

722 tuning during the development of contra-and ipsilateral wirings. In the contralateral

723 pathway, the RGC mosaic initially seeds the cortical map structure of orientation

724 preference. In the ipsilateral pathway, the initial map is seeded by the ipsilateral

725 RGC mosaics, independent of contralateral map structure. Next, during the 
726 matching process by activity-dependent plasticity, the connections from ipsilateral

727 pathways are refined to match the orientation preference of the contralateral

728 map $^{23}$. c, Difference of preferred orientations between the two pathways before

729 and after binocular matching. 
731 Supplementary Figure 2. Stripe pattern of ocular dominance can be dominance map is distorted so that the blobs of monocular regions are merged into stripe patterns of ocular dominance.

d, As in our model simulation, in c, the stripe a

b

Ocular dominiance map RGC (Visual space)

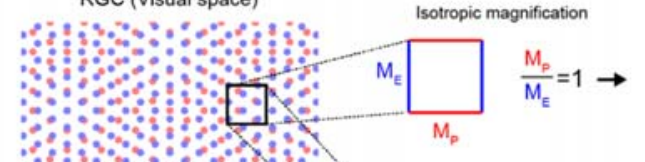

c

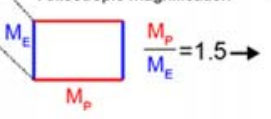

d

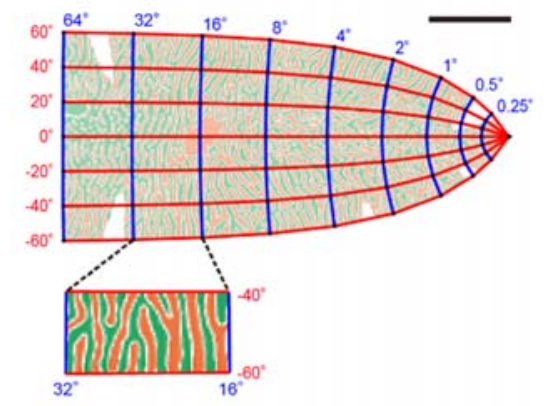
pattern of ocular dominance appeared to be parallel to the direction of shorter magnification, which is consistent with the direction of iso-eccentricity in monkeys ${ }^{1}$. 
a

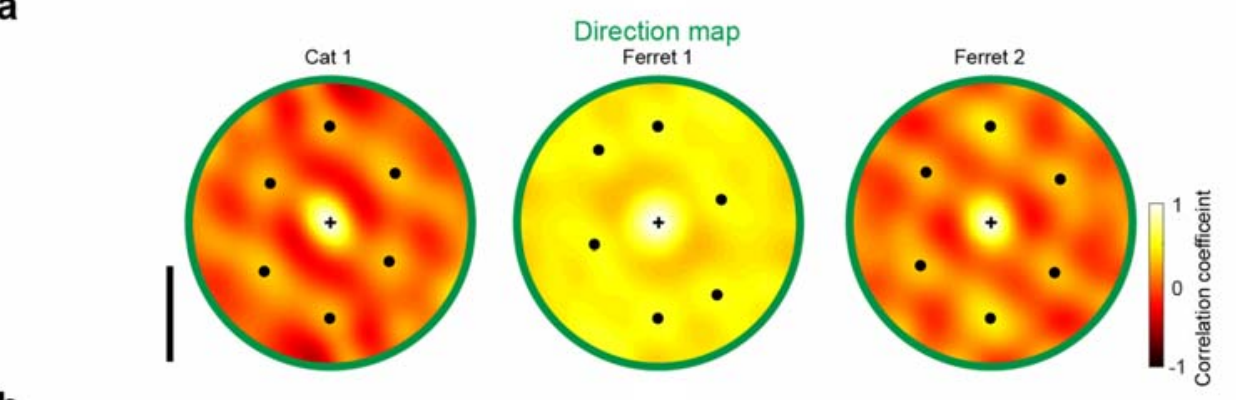

b
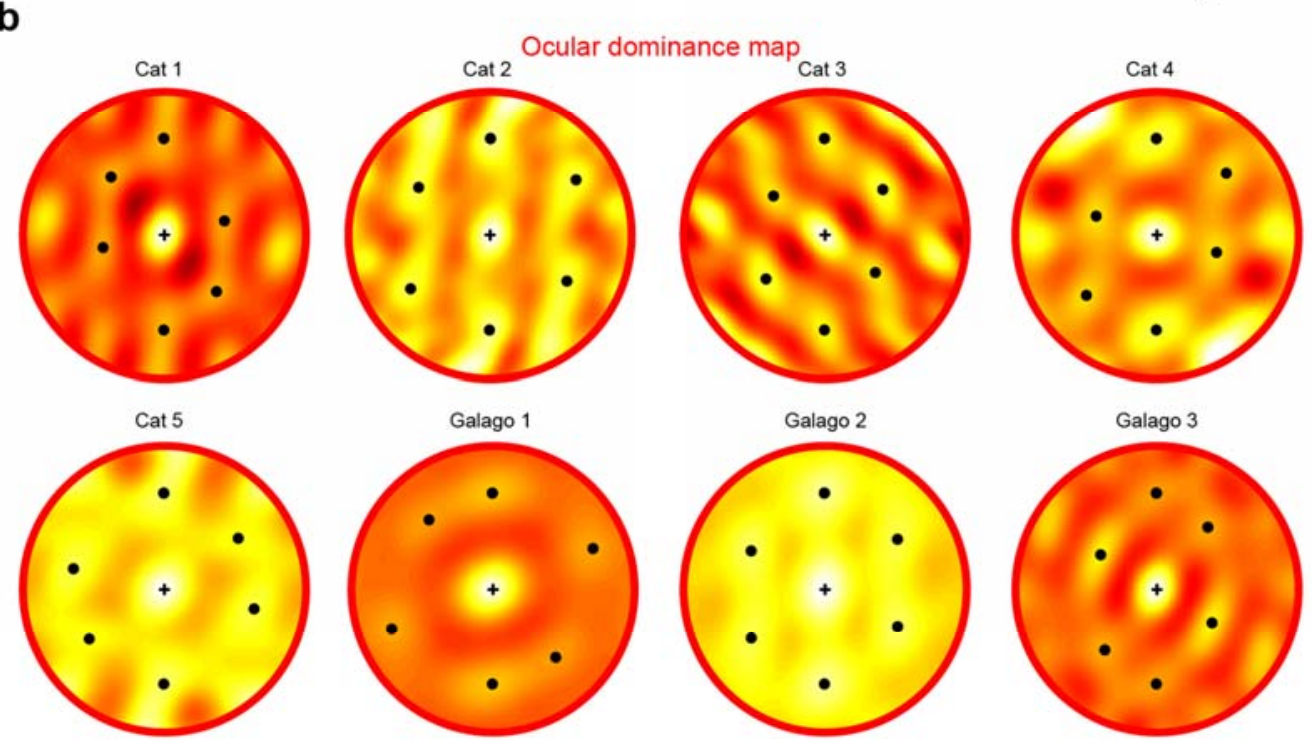

C
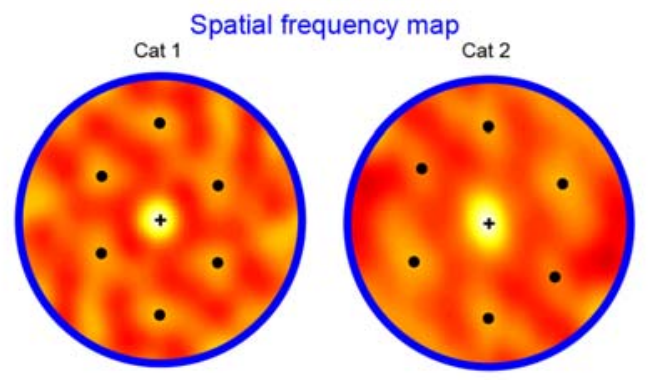

740 Supplementary Figure 3. Hexagonal pattern of individual functional maps

741 Hexagonal pattern observed in the autocorrelation of individual functional maps.

742 The magnitudes of local maxima (solid black dots) are statistically significant

743 (bootstrap analysis, $P<0.01$ ). a, Direction maps in cats ${ }^{27}$ and ferrets ${ }^{29,30} . \mathbf{b}$, Ocular 
bioRxiv preprint doi: https://doi.org/10.1101/354126; this version posted June 22, 2018. The copyright holder for this preprint (which was

not certified by peer review) is the author/funder. All rights reserved. No reuse allowed without permission.

744 dominance maps in cats c $^{7,10,25,28}$ and galagos ${ }^{26}, \mathbf{c}$, Spatial frequency maps in

745 cats $^{7,25}$. 